\title{
- El sistema político brasileño y las elecciones de 2018: Bolsonaro y el cambio de actores en la política nacional
}

\author{
Lucas Arantes Miotti*
}

\section{Introducción}

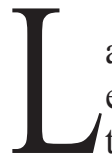

as elecciones presidenciales de 2018 tuvieron una fuerte influencia en el cambio de escenarios en la política nacional de Brasil, que representó la pérdida de poder de los partidos políticos tradicionales, así como la ascensión de nuevos actores en el juego político. Este es un escenario de análisis interesante, porque termina con prácticamente toda la lógica política vigente en los últimos 15 o 20 años.

La elección de Fernando Collor en 1989, fue la primera posterior a la dictadura militar realizada con voto directo. En esta ocasión el Partido de los Trabajadores (PT) y el Partido de la Social Democracia Brasileña (PSDB) no se disputaron para ocupar la presidencia del país. A partir de 1989, todas las elecciones para presidente tuvieron como principales candidatos a los del PT y del PSDB; sin embargo, esta lógica se fraccionó en 2018 con la llegada al poder de Jair Bolsonaro del Partido Social Liberal (PSL).

Por otra parte, hubo una importante renovación de los representantes en los grupos legislativos del país, que provocó la presencia de más partidos políticos en el juego legislativo, junto con una importante pérdida del poder de los grandes partidos políticos de Brasil: PT, PSDB y Movimiento Democrático Brasilero (MDB). Estas características son importantes para reflexionar sobre

Licenciado en Ciencia Política y Sociología, Universidad Federal de la Integración Latinoamericana, Brasil. Magíster en Estudios Latinoamericanos, Universidad Andina Simón Bolívar, Sede Ecuador; 〈lucas.miotti@hotmail.com〉. 
la estructura de la "nueva" política nacional brasileña y, principalmente, cuál será el panorama que tendrá para gobernar el nuevo presidente electo, Jair Bolsonaro.

En este trabajo se utilizó el método de análisis histórico comparativo, que es útil para identificar los patrones, mecanismos y dinámicas de la política de un país o de sus estructuras organizacionales. ${ }^{1}$ El indicador que sirve de base a la investigación es la composición de la Cámara de Diputados, del Senado Federal y los partidos que controlan los ejecutivos de los estados brasileños, que permite observar de forma más específica los cambios suscitados en cada elección.

En el artículo se analizan las tres últimas elecciones nacionales de Brasil, que representan un constante descenso de los actores políticos tradicionales, hasta el surgimiento de nuevos actores, que se discute en detalle a lo largo del trabajo.

Además, se realiza un recuento sobre los principales candidatos a la Presidencia de la República desde la democratización del país, para observar de mejor manera el argumento del trabajo sobre la "hegemonía" de los partidos tradicionales. El proceso de recuento histórico comparativo permite tener una noción sobre cómo estuvo estructurada la política de Brasil, lo que sirve de base para demostrar la significación de las elecciones de 2018.

El trabajo se encuentra dividido en tres partes. En la primera parte, se discutirá exclusivamente sobre el sistema político brasileño, para explicar cómo funciona el manejo político del país, la importancia de cada esfera de poder y de los roles que cumple cada uno de los actores políticos. La segunda parte, expone la situación de ruptura de la lógica electoral de los últimos años, que refleja el cambio en las elecciones de 2018, en comparación con las elecciones anteriores, para demostrar la transformación de los actores en la política brasileña. Finalmente, la tercera parte analizará la política del nuevo presidente electo, Jair Mesías Bolsonaro, a través de su plan de gobierno, para construir un panorama sobre cómo se direccionará la política de Brasil en los próximos años.

1. Helder Ferreira do Vale, "Temporality, causality and trajectories: comparative historical analysis in social and political sciences", Revista Debates 9, No. 1 (2015): 61-87. 


\section{El sistema político brasileño}

En esta sección se debaten varios aspectos sobre el sistema político brasileño estructurado en el presidencialismo de coalición, que se enfoca en sus compilaciones institucionales y organizativas, para demostrar las estructuras y el funcionamiento de la toma de decisiones dentro de este sistema. Brasil adoptó un modelo presidencialista, gobernado en coalición, federativo, con sistema de elección proporcional, mayoritario y multipartidario; como una manera para reducir las disparidades sociales y los conflictos.

El presidencialismo tiene características muy específicas sobre su modo de accionar. El presidente es electo por voto popular, los mandatos tanto del poder ejecutivo como del legislativo no dependen de la confianza mutua, ya que el presidente, entre otras facultades, nombra, dirige la composición del gobierno y tiene poderes legislativos constitucionales. ${ }^{2}$ La Federación es un sistema de descentralización de la política, que otorga autonomía a núcleos de poder político -en el caso de Brasil a los estados-. Además, en la lógica de la Federación, cada estado miembro posee representaciones en el Congreso Nacional del Brasil, en el marco de la política nacional.

El sistema de elección brasilero combina una lógica proporcional, utilizada en la Cámara de Diputados y en esferas legislativas de estados y municipios; y una lógica mayoritaria, utilizada para los cargos ejecutivos del país y el Senado Federal, donde los candidatos con mayor número de votos son electos. En el sistema de lista abierta, cada partido obtiene un número de representantes proporcional a la suma de votos de todos sus candidatos, y los representantes se definen por el candidato que obtuvo más votos en el partido. ${ }^{3}$

Por último, Brasil adoptó un sistema de partidos multipartidario, caracterizado por la existencia de tres o más partidos que pueden estar a cargo del ejecutivo nacional por medio de una coalición. No obstante, el sistema está marcado por una gran fragmentación, debido a la gran cantidad de partidos participantes en el esquema político nacional. ${ }^{4}$

2. Giovanni Sartori, Engenharia constitucional: como mudam as constituições (Brasília: UnB, 1996); Vicente Palermo, "¿Cómo se gobierna Brasil? El debate brasileño sobre instituciones políticas y gestión de gobierno", Desarrollo Económico 40, No. 159 (2000): 493-518.

3. Manoel Rodrigues Ferreira, A evolução do sistema eleitoral brasileiro (Brasilia: Tribunal Superior Eleitoral, 2005), edición eBooksBrasil, 〈https://bdjur.tjce.jus.br/jspui/handle/123456789/150〉.

4. Palermo, “Cómo se gobierna Brasil? El debate brasileño sobre instituciones políticas y gestión de gobierno". 
El modelo se consideraba como un sistema inestable. Sergio Abranches, uno de los principales autores que defienden este argumento señala que este sistema es de alto riesgo porque se sustenta en la capacidad de negociación que tiene el poder ejecutivo y en el respeto de los temas considerados como no negociables, que no son siempre explícitos al momento de formar la coalición. ${ }^{5}$

Con el pasar de los años estas concepciones fueron olvidadas, ya que en la práctica el sistema no fue completamente inoperante, a pesar de los dos impeachments posteriores a la redemocratización. Así, la característica central del presidencialismo brasileño se formó en torno a la coalición, un modelo en que el ejecutivo pierde la centralidad unipersonal del presidente, de esta forma se distribuye el poder de decisión entre los miembros de la coalición. Es exactamente esta distribución de poder que hace a la coalición importante. ${ }^{6}$

Es fundamental resaltar que el sistema de coalición se establece para que el gobierno logre una mayoría en el Congreso Nacional, y de esta forma adquiera estabilidad. Esto es necesario por el esquema de composición del Congreso, en el que sería prácticamente imposible que el partido del presidente establezca una mayoría.

El Congreso brasilero es bicameral, compuesto por una Cámara de Diputados y un Senado. La Cámara de Diputados del Brasil cuenta con 513 representantes repartidos entre los estados y el Distrito Federal de manera proporcional a sus electorados. Mientras que en el Senado Federal está constituido por 81 representantes, que equivale a tres representantes por cada estado y distrito.

En la actualidad se encuentran registrados 35 partidos políticos en el Tribunal Superior Electoral (TSE), que en la práctica hace imposible que un partido logre la mayoría en ambas instancias del Congreso Nacional, y da como resultado que la coalición sea el medio para que el partido de gobierno logre una mayoría que le permita tener un mayor nivel de gobernanza en el Estado.

El poder ejecutivo se encuentra en una posición estratégicamente favorable para negociar con los parlamentarios y sus partidos, así está en capacidad

5. Sergio Henrique Abranches, "Presidencialismo de coalizão: O dilema institucional brasileiro", Revista de Ciências Sociais 31, No. 1 (1988): 5-34.

6. Vicente Palermo, "Brazilian political institutions: an Inconclusive Debate", Brazilian Political Science Review 10, No. 2 (2016): 1-29. 
de explorar estratégicamente el control que tiene sobre los bienes relacionados con el ejercicio del poder para reducir los "costos" del apoyo recibido. Como el presidente obtiene el monopolio para el acceso a los recursos públicos, él mismo puede obtener ventajas estratégicas para la formación de su coalición en el gobierno. ${ }^{7}$

Limongi y Figueiredo argumentan que si el presidente está dotado de poderes legislativos, como en el caso de Brasil, es capaz de imponer una agenda legislativa de trabajo para inducir a los parlamentarios a la cooperación. Otro factor clave es el control que el presidente tiene para acceder a los puestos del gobierno. Si los parlamentarios quieren tener participación directa en el gobierno, deben sacrificar su autonomía en la formulación de políticas públicas.

Por lo tanto, es posible argumentar que el sistema político brasilero funciona a través de un presidente fuerte, con grandes capacidades de negociación. Sin embargo, existen varias estrategias utilizadas por el presidente para lograr la cooperación de los parlamentarios, bajo el principio de que sus agendas son divergentes.

Algunos argumentos aseguran que un presidente fuerte no necesita cooperación del Congreso, ya que por su propia fortaleza impone su voluntad. Sin embargo, el incentivo a la negociación surge, de cierta manera, de las herramientas proactivas del presidente, pero principalmente de los equilibrios institucionales entre los actores que son inducidos a cooperar. Su capacidad de gestión resulta ser más dependiente de la capacidad del presidente en organizar la cooperación, que de sus recursos para imponer su agenda. ${ }^{8}$

La frecuencia de las coaliciones refleja la fragmentación partidario-electoral, que está vinculada a las diferencias socioculturales. Las sociedades más divididas y más conflictivas requieren de la formación de alianzas y una mayor capacidad de negociación para la gobernabilidad y la estabilidad institucional.

Si bien el presidente tiene grandes poderes legislativos, en caso de que quiera gobernar solo, su gobierno estará destinado al fracaso. Esto ocurre por el alto número de miembros del legislativo que buscan acceder a diferentes

7. Fernando Limongi y Argelina Figueiredo, "Bases institucionais do presidencialismo de coalizão", Lua Nova, No. 44 (1998): 87-106.

8. Palermo, "Brazilian Political Institutions: An Inconclusive Debate"; Mattew Shugart y John Carey, Presidents and Assemblies: Constitutional Design and Electoral Dynamics (Cambridge: Cambridge University Press, 1992). 
cargos en el gobierno, por lo que deben negociar con el ejecutivo para acceder a esos puestos. ${ }^{9}$

Configurar la coalición no significa restringir el poder del presidente, ya que la propia coalición permite que su poder sea efectivo. El presidente anticipa que sin la coalición para obtener una mayoría en el Congreso, será prácticamente imposible aprobar cualquier proyecto de su agenda de gobierno. ${ }^{10}$

Sergio Abranches discute un punto relevante sobre la lógica de la coalición que demuestra cómo funciona el juego de la política en la Federación de Brasil. El autor argumenta que la lógica de formación de las coaliciones cuenta con dos ejes: el partidario y el regional/estadual, por las grandes coaliciones que son realizadas por medio de un cálculo relativo a la base de sustentación política del gobierno, que no es solamente partidario-parlamentario, sino también regional o estadual, lo que demuestra la importancia que los estados tienen para la política nacional.

La gobernabilidad depende también de la capacidad de negociación para la composición regional y partidaria de los ministerios. En estas entidades las fuerzas de los Estados se constituyen en agentes que pueden presentar problemas para la gobernabilidad del país. ${ }^{11}$ La conformación de la coalición no se plantea solo en función del Congreso Nacional, sino en la conformación política de los Estados de la Federación.

El análisis realizado sobre el sistema político brasilero nos permite observar de manera más efectiva los resultados de las elecciones de 2018 en Brasil, para discutir aspectos más específicos como son: la pérdida de poder de los partidos tradicionales y el ascenso de nuevas fuerzas a la política nacional; con el objetivo de observar el rol de cada actor en el juego político del país en el nuevo gobierno.

9. Sergio Henrique Abranches, "Presidencialismo de coalizão: O dilema institucional brasileiro"; Fernando Limongi y Argelina Figueiredo "Bases institucionais do presidencialismo de coalizão".

10. Andréa Freitas, O presidencialismo da coalização (Rio de Janeiro: Fundação Konrad Adenauer, 2016).

11. Octavio Amorim, "A governabilidade sob a presidência de Lula", Revista Conjuntura Econômica 57, No. 4 (2003): 22-8. 


\section{El fin de la "hegemonía" y el ascenso de nuevos actores en la política nacional}

En esta sección se describen los cambios operados por la elección de 2018 en la política nacional de Brasil. A través de un análisis histórico, se tratará sobre la conclusión de la "hegemonía" entre el PT y el PSDB por la disputa presidencial, así como el ascenso de nuevos actores en la política nacional en la Cámara de Diputados, Senado Federal y en los ejecutivos de cada Estado de la Federación, para establecer la composición de la nueva política de Brasil a partir de 2019, y realizar un análisis posterior sobre los aspectos de gobernabilidad.

En 1994 durante la segunda elección presidencial posterior a la dictadura militar, el PT y el PSDB se presentaron como principales protagonistas a la disputa de la Presidencia de la República. En esta ocasión resultó vencedor el expresidente Fernando Henrique Cardoso del PSDB, quien dirigió el país hasta el año 2002 en que entrega la presidencia a Lula da Silva. A continuación se observa cuáles fueron los dos principales partidos en las elecciones directas para presidente desde el retorno a la democracia.

- Tabla 1. Candidatos y electos en las elecciones presidenciales de Brasil (1989-2018)

\begin{tabular}{|c|c|c|}
\hline Año de elecciones & Candidatos (Partido Político) & Candidato electo \\
\hline 1989 & $\begin{array}{l}\text { Fernando Collor (PRN) } \\
\text { Luis Inácio Lula da Silva (PT) }\end{array}$ & Fernando Collor (PRN) \\
\hline 1994 & $\begin{array}{l}\text { Fernando Henrique Cardoso (PSDB) } \\
\text { Luis Inácio Lula da Silva (PT) }\end{array}$ & Fernando Henrique Cardoso* (PSDB) \\
\hline 1998 & $\begin{array}{l}\text { Fernando Henrique Cardoso (PSDB) } \\
\text { Luis Inácio Lula da Silva (PT) }\end{array}$ & Fernando Henrique Cardoso* (PSDB) \\
\hline 2002 & $\begin{array}{l}\text { Luis Inácio Lula da Silva (PT) } \\
\text { José Serra (PSDB) }\end{array}$ & Luis Inácio Lula da Silva (PT) \\
\hline 2006 & $\begin{array}{l}\text { Luis Inácio Lula da Silva (PT) } \\
\text { Geraldo Alckmin (PSDB) }\end{array}$ & Luis Inácio Lula da Silva (PT) \\
\hline 2010 & $\begin{array}{l}\text { Dilma Rousseff (PT) } \\
\text { José Serra (PSDB) }\end{array}$ & Dilma Rousseff (PT) \\
\hline 2014 & $\begin{array}{l}\text { Dilma Rousseff (PT) } \\
\text { Aécio Neves (PSDB) }\end{array}$ & Dilma Rousseff (PT) \\
\hline 2018 & $\begin{array}{l}\text { Jair Bolsonaro (PSL) } \\
\text { Fernando Haddad (PT) }\end{array}$ & Jair Bolsonaro (PSL) \\
\hline
\end{tabular}

* Electo en primera vuelta.

Fuente: Brasil Tribunal Superior Electoral, "Repositório de dados eleitorais".

Elaboración: autor. 
De esta manera, se puede observar que en todas las elecciones directas a la Presidencia de la República posteriores al retorno a la democracia, el PT estuvo entre los dos candidatos más votados, por lo que ganó la mitad de las elecciones de este periodo. Asimismo, se observa que en dos elecciones el PT y el PSDB no se disputaron el primer y segundo lugar para la presidencia: el primero en el año 1989, con la elección de Fernando Collor, y el segundo en el año 2018, con la elección de Jair Bolsonaro.

Las últimas elecciones presidenciales marcaron un hito importante en el país, en vista de que en los últimos veinte años el PT y el PSDB se habían consolidado como los principales partidos políticos en disputa por la Presidencia de la República. Las elecciones de 2018 terminaron con esta "hegemonía", en el momento en que Jair Bolsonaro surgió como presidente electo, con un partido que nunca antes había llegado tan lejos en un proceso electoral.

Además, la elección representó una nueva configuración en el poder legislativo brasileño, un escenario en donde los partidos tradicionales perdieron representación y nuevos actores aparecieron en la disputa política. El caso que más ejemplifica esta situación es el PSL, partido liderado por Bolsonaro, presidente electo del país; que llegó a ocupar en estas elecciones 52 puestos en la Cámara de Diputados, mientras que en las elecciones del año 2014 logró conseguir apenas un solo puesto.

Por otro lado el MDB, partido político del expresidente Michel Temer, caracterizado por ser uno de los más grandes del Brasil y por estar siempre presente en los gobiernos desde el regreso a la democracia, sufrió un infortunio en las urnas. En las elecciones del año 2014 el partido logró conquistar 66 plazas en la Cámara de Diputados, mientras que en esta elección logró tener apenas 34 representantes.

Los partidos tradicionales como el PSDB y el PT también se vieron afectados en las urnas. El PSDB obtuvo 54 representantes para la cámara en las elecciones de 2014; sin embargo, en la última elección su número se redujo a casi la mitad, con apenas 29 representantes. El PT, pese a lograr en estas elecciones el mayor número de representantes en la Cámara de Diputados, sufrió de igual manera una disminución en comparación con las elecciones anteriores. En 2014, el partido consiguió 69 representantes, mientras que en 2018 solamente obtuvo 56 puestos. 
El gráfico 1 establece el número de representantes que obtuvieron el PT, el PSDB y el MDB, junto con el PSL en la Cámara de Diputados, con el propósito de analizar el crecimiento que tuvo este último partido en las elecciones de 2018, en comparación con la evidente pérdida de poder de los tres partidos tradicionales de Brasil.

- Grafico 1. Número de representantes del PT, PSDB, MDB y PSL en la Cámara de Diputados en las tres últimas elecciones

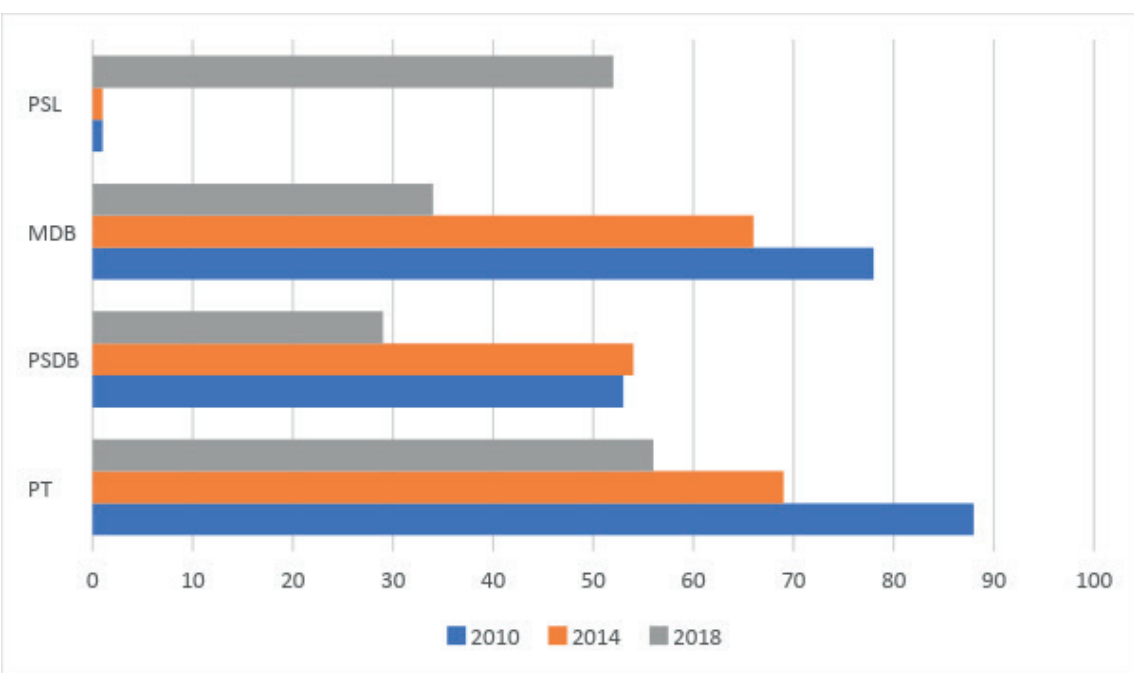

Fuente: Brasil Tribunal Superior Electoral, "Partidos políticos registrados no TSE".

Elaboración: autor.

El gráfico establece un constante descenso en la representación de los tres grandes partidos y, a su vez, un gran aumento de representatividad del PSL, que se configura en las últimas elecciones. No obstante, las pérdidas que han sufrido los partidos tradicionales no se deben al aumento de votación que obtuvo el PSL, sino también a la inserción de nuevos partidos con representación en la Cámara de Diputados. En 2010 hubo 22 partidos políticos con representantes en la Cámara de Diputados, para 2014 el número se incrementó a 28 partidos representados y, en la actualidad, son 30 partidos políticos los que ocupan esa representación.

El incremento de partidos se suma al porcentaje de renovación en la casa legislativa. Según datos de la Cámara de Diputados, la elección de 2018 tuvo 
un $47 \%$ de nuevos representantes, porcentaje que es similar al de los dos primeros años de redemocratización, pero poco común al de los últimos veinte años.

- Grafico 2. Porcentaje de renovación de la Cámara de Diputados por año de elección

\section{A renovação da câmara}

Deputados novos (primeiro mandato) a cada legislatura

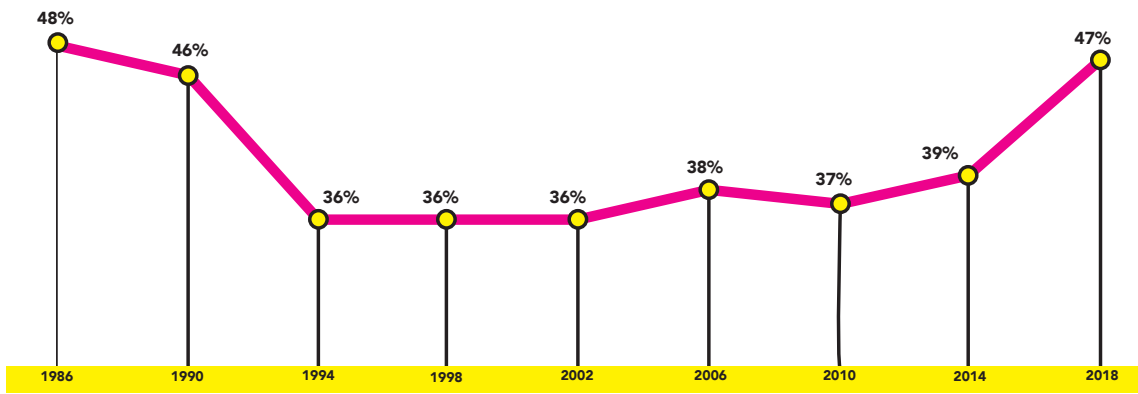

Fuente: Ana Cristina Campos, "Taxa de renovação da Câmara dos Deputados foi a maior em 20 anos", Agência Brasil, 2018.

Además, al momento de observar la configuración del Senado federal, se puede evidenciar que hubo de igual manera una renovación con pérdida de fuerza de los partidos tradicionales. El partido con mayor número de representantes en el Senado es el MDB, con 12 senadores, a pesar de haber perdido 6 senadores frente a las elecciones anteriores. Lo mismo aconteció con el PSDB, con 8 representantes, con una pérdida de 4 en relación a la elección anterior y el PT ahora con 6 senadores, que pierde 3 desde las elecciones de 2014.

El gráfico 3 establece la comparación del número de representantes en el Senado Federal de los partidos tradicionales con el PSL, durante las últimas tres elecciones. Se puede percibir que la representación en el Senado ha mantenido una misma lógica en los últimos años, similar a la Cámara, pero con una simple diferencia. Los tres partidos tradicionales demuestran de igual manera, un continuo descenso en las tres últimas elecciones, la diferencia en este caso fue que el partido del presidente electo no logró conseguir tanta representatividad como en la Cámara de Diputados. 
- Grafico 3. Número de representantes del PT, PSDB, MDB y PSL en el Senado Federal en las últimas tres elecciones

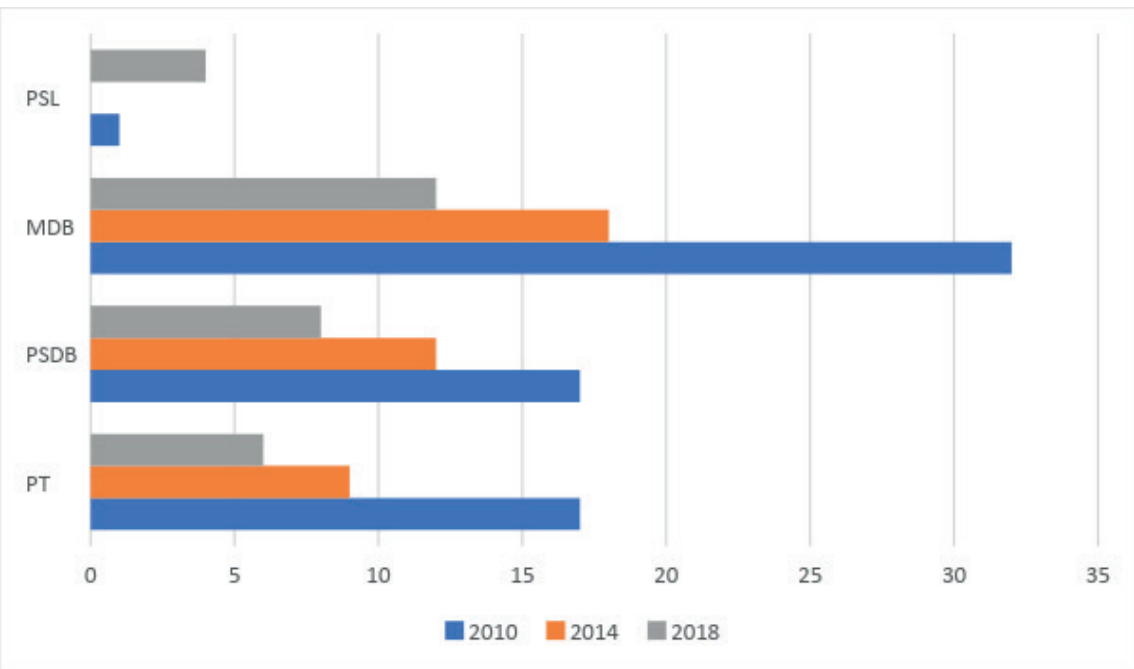

Fuente: Brasil Tribunal Superior Electoral, "Partidos políticos registrados no TSE".

Elaboración: autor.

El mismo escenario se presenta cuando se analizan las elecciones para gobernadores de los Estados de Brasil, debido a que partidos considerados como pequeños lograron llegar al ejecutivo de algunos estados, entre los que se encuentran el PSL, el Partido NOVO y el Partido Social Cristiano (PSC). Estos partidos se apoyaron en las ideas de Jair Bolsonaro, sin la necesidad de apoyar directa y abiertamente al candidato. El PSL logró conquistar el ejecutivo de tres estados: Santa Catarina, Roraima y Rondonia, mientras que el partido NOVO logró por su parte conquistar el estado de Minas Gerais, y por último, el PSC conquistó al estado de Río de Janeiro y al estado del Amazonas.

Esto demuestra un pequeño pero significativo ascenso de otros partidos en el juego político de la Federación brasileña, para conquistar estados con una gran población como el caso de Río de Janeiro y Minas Gerais. Sin embargo, el partido que más eligió gobernadores de los estados fue el PT que obtuvo cuatro representaciones. En el gráfico 4 se puede observar de forma más sencilla el número de Estados con los que cuenta cada partido. 
- Grafico 3. Número de estados conquistados por partidos políticos en las elecciones de $2018^{12}$

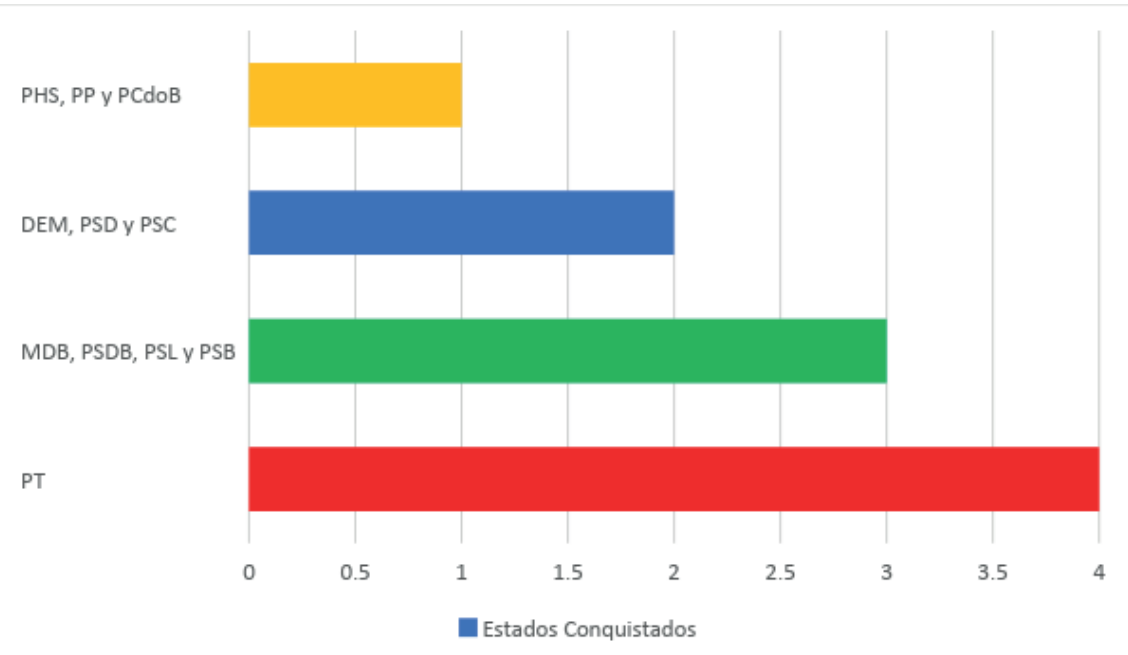

Fuente: Brasil Tribunal Superior Electoral, "Estatísticas eleitorais".

Elaboración: autor.

Este escenario establece el cambio de tendencia en el juego político nacional presentado en las últimas elecciones, que debilitó a los grandes partidos tradicionales como el PT, el PSDB y el MDB. A pesar de ello, estos partidos mantienen su relevancia dentro de la política nacional. El PT por ejemplo obtuvo el mayor número de Estados y diputados en la Cámara, pese a haber perdido la disputa por la presidencia del país.

A partir de este momento el partido de gobierno tendrá que relacionarse y negociar con los partidos tradicionales para poder adquirir gobernabilidad, ya que el debilitamiento de los partidos tradicionales no resultó en la pérdida del poder político, pero si en una reducción en su tamaño e influencia. Es así, como la lógica del sistema político de Brasil se estructura en un presidencialismo de coalición, en donde para que el nuevo presidente pueda gobernar,

12. Nombres de los partidos no mencionados anteriormente: Partido Humanista de la Solidaridad (PHS), Partido Progresista (PP), Partido Comunista de Brasil (PCdoB), Demócratas (DEM), Partido Social Democrático (PSD) y Partido Socialista Brasilero (PSB). 
deberá relacionarse con los partidos que cogobiernan. En este escenario, se puede observar una tarea compleja por lograr que es la gobernabilidad, ya que existe una política más fragmentada que en los últimos años, con más actores con poder relativo.

\section{La política de Jair Mesías Bolsonaro}

La política de Brasil sufrirá una transformación a partir del año 2019. Esta transformación llegó a prácticamente todas las esferas del poder político del país, como resultado de un discurso fuerte y polémico del nuevo presidente electo, Jair Mesías Bolsonaro, por lo que es necesario discutir sobre la postura del presidente en campaña y las principales políticas de su plan de gobierno.

El presidente electo estuvo presente en la política nacional desde el año 1990, cuando fue electo por primera vez como diputado federal. Bolsonaro permaneció en ese puesto hasta las elecciones de 2018. Inspirado en la incredulidad de la población en la política, Bolsonaro lanzó su candidatura como la persona que iba romper con la política tradicional pervertida por la corrupción. Una de sus frases más simbólicas durante toda la campaña fue: "voy a acabar con todo eso ahí", con esta frase simple y corta se refiere principalmente a la corrupción en el sistema político del país.

A pesar de la corta campaña, marcada por un atentado a su persona y por rehusarse a participar en debates políticos, Bolsonaro emitió frases polémicas y hasta con cuño antidemocrático. Conducido por el "antipetismo", ${ }^{13}$ el candidato profirió un discurso en el estado de Acre, en el que de manera exaltada dijo: "vamos a fusilar a todos los petistas (...) y expulsarlos de acá", refiriéndose a las personas que son simpatizantes del PT. Toda su campaña estuvo basada en el odio e indignación a los gobiernos del PT y a sus simpatizantes. De esta manera, ¿Qué se puede esperar de la política del nuevo presidente?

Desafortunadamente en el plan de gobierno del presidente electo no está claro cuáles van a ser sus acciones para temas clave como son el combate a la corrupción, la educación y la economía. No obstante, pueden sacarse algunas conclusiones sobre cómo será su política. El título de su plan de gobierno es

13. Este fue el término utilizado en Brasil para todo discurso o movimiento que era contrario al PT. 
Brasil acima de tudo, Deus acima de todos, ${ }^{14}$ demostrando desde un inicio el vínculo que tiene su gobierno con el cristianismo, del que Bolsonaro busca retomar los valores y la ética de la familia. Dentro de su plan consta una política que también configuró como un fuerte distintivo de su campaña, el liberalismo.

En su plan de gobierno consta el liberalismo como la manera para disminuir la crisis económica del país, disminuir el desempleo y retomar el crecimiento económico. Sobre la base del liberalismo, Bolsonaro pretende reducir al Estado y a la burocracia, para otorgar mayor autonomía al mercado nacional. Más específicamente, buscará privatizar determinados sectores del Estado que el gobierno no considere importantes, así como reducir el número de ministerios del país.

El principal eje de su plan de acción es la seguridad pública, que tiene dos vertientes importantes para el futuro de su gobierno. El primero es mantener a los criminales por un tiempo más prolongado en las cárceles, a través de un sistema penitenciario más rígido. El segundo es la revocación de la ley de desarme, para flexibilizar la tenencia de armas en el ciudadano común, como un medio para inhibir la acción criminal. Bolsonaro argumenta que esta será su primera acción cuándo sea presidente.

Otro tema importante es cómo planea manejar las relaciones internacionales de Brasil. El plan demuestra que el gobierno buscará retomar relaciones con países que aporten al desarrollo y que fueron descartados anteriormente por cuestiones ideológicas. Principalmente, buscará enfocarse en establecer relaciones bilaterales beneficiosas para Brasil, en valor y en desarrollo tecnológico. Bolsonaro, durante su campaña, criticó la acción de China y se refirió a Venezuela de manera negativa, país al que puso como ejemplo de la acción de gobiernos en manos de partidos como el PT.

Bolsonaro no se preocupará por bloques como los BRICS, la UNASUR o el MERCOSUR, para convertirse en un gran "enemigo" regional de países que tengan ideologías totalmente contrarias a la suya, como Venezuela o Bolivia. Dentro de la aspiración para establecer relaciones bilaterales se espera una mayor aproximación a Estados Unidos, en detrimento de sus relaciones con China, debido a que el plan de gobierno determina que el presidente

14. Texto traducido: "Brasil arriba de todo, Dios arriba de todos". 
buscará consolidar relaciones con "países de democracias fuertes", ${ }^{15}$ como Estados Unidos e Israel, alejándose de una política de bloques regionales o interregionales.

En caso de que los tres ejes principales del plan Bolsonaro en materia de economía, seguridad y relaciones internacionales sean concretados, se construirá un escenario completamente diferente al que se ha observado durante el comienzo del siglo XXI. Brasil se abrirá más hacia el comercio global con una política exterior basada en las relaciones bilaterales. Además de la implementación de una política de seguridad relativiza a los derechos humanos ya que, según el propio presidente electo, solo defienden a los criminales, mientras que las víctimas quedan desprotegidas.

Pero, para que todo esto pueda cumplirse, el Presidente tendrá que adquirir una capacidad de gobernar por medio de una coalición, como ya se mencionó anteriormente. La coalición del gobierno podrá visualizarse el momento en que se anuncien oficialmente los encargados de cada ministerio, que es una poderosa herramienta para conseguir el apoyo de otros partidos. El tiempo mostrará los próximos pasos del nuevo presidente, Jair Mesías Bolsonaro, dentro de esta nueva era conservadora en principios, y liberal en términos econométricos.

\section{Consideraciones finales}

Como se analizó a lo largo del artículo, el sistema político brasileño se fundamenta en la capacidad de negociación del poder ejecutivo con los miembros del Congreso Nacional y con otros partidos, debido a que el gobierno debe conformar una coalición para adquirir la capacidad de gobernar. De esta manera, surge la pregunta: ¿Qué se puede esperar de la política de Brasil para los próximos años?

La primera cuestión destacada en el trabajo de investigación, es la difícil tarea del ejecutivo de negociar con el poder legislativo más numeroso de la historia. Es y ha sido el más grande por el aumento de partidos que lograron conseguir representantes en las dos esferas del legislativo brasileño; que cons-

15. Partido Social Liberal, "Brasil acima de tudo, Deus acima de todos: o caminho da prosperidade." Propuesta de Plan de Gobierno, Brasilia, 2018. 
tituye un mayor trabajo para el jefe del ejecutivo, por el hecho de que tendrá una mayor cantidad de actores con quienes negociar la implementación de sus políticas.

Este análisis no toma en consideración el aspecto de la disciplina partidaria, porque asume que los partidos tienen el control de sus representantes y que, además, respetan las directrices del partido. En casos de indisciplina partidaria, que son frecuentes en partidos políticos pequeños, la gobernabilidad se tornará más difícil porque el ejecutivo se verá en la obligación de negociar con cada uno de los representantes y no con el partido.

Sin embargo, un punto importante sobre la gobernabilidad es que algunos candidatos que fueron elegidos, mantienen tendencias similares a la del presidente electo, como la tenencia de armas de fuego para resguardar la seguridad o un discurso fuerte contra la corrupción, sin que de forma expresa declararan su apoyo público durante la campaña del candidato Bolsonaro, lo que sería un punto a favor del presidente electo al contar con sectores del Congreso y de la población afines a sus posiciones.

Sin duda el gobierno de Bolsonaro tendrá que buscar apoyo en uno o más partidos tradicionales como el PT, el PSDB o el MDB. En este contexto, es evidente que el PT será el principal partido opositor al nuevo gobierno, porque la campaña política de Bolsonaro estuvo basada en el odio y en el desprestigio a los gobiernos de este partido.

Por otra parte, se espera que el MDB sea un partido que complemente al actual gobierno, al ser un partido históricamente fragmentado, con diversas facciones en su interior, pero que mantiene como líder al expresidente Michel Temer, un político que ha demostrado tener ideologías similares a las de Bolsonaro, lo que podría facilitar una alianza. Además, el MDB es un partido estratégico para el gobierno, porque cuenta con un número significativo de representantes en la Cámara de Diputados y de gobernadores de estados, con el mayor número de senadores.

Lo que diferencia al PSDB con los otros dos partidos políticos es que se encuentra en una fragmentación bipolar, originada por el surgimiento de nuevas fuerzas políticas en su interior, como el gobernador electo de São Paulo, João Doria, en detrimento del fracaso electoral del presidente del partido Geraldo Alckmin, quien no consiguió llegar a la segunda ronda de las elecciones presidenciales. 
João Doria en su campaña llegó a declarar su apoyo a Bolsonaro, a pesar de que su partido, comandado por Alckmin no hizo lo mismo. En estos casos el apoyo o no al gobierno dependerá de la resolución de la lucha política interna del partido, por lo que se espera que el PSDB tenga una postura inicial de centro para apoyar o no al gobierno.

Al momento de analizar lo que puede ser la política del nuevo gobierno, lo más seguro es que retorne al modelo neoliberal que busca reducir la maquinaria estatal, proporcionar una mayor libertad al mercado y privatizar las empresas públicas. Estos tres temas fueron ejes fundamentales durante la campaña del presidente electo.

Además, Bolsonaro cuenta con un alto índice de popularidad, que le permite poner en discusión temas polémicos como la reforma de la previsión social o la tenencia de armas. Sin embargo, se espera una postura menos radical del ejecutivo en términos políticos de la que se planteaba durante el periodo de campaña.

En lo que respecta a los temas económicos, resulta claro que buscará direccionar al país hacia un camino diferente al del gobierno precedente. Sin embargo, el presidente tendrá que negociar continuamente y ceder posiciones para ejercer su gobierno, lo que resulta en cierta medida que su forma radical e impositiva sea inhibida por una acción negociadora.

Finalmente, lo que se puede esperar para el futuro de este gobierno es una encrucijada política. Bolsonaro fue electo por su postura radical e impositiva, contraria a la política tradicional, sin embargo, tiene la necesidad de convertirse en un ente negociador con los propios partidos tradicionales, para adquirir las condiciones necesarias que le permitan gobernar. Esta encrucijada puede ocasionar la pérdida de su popularidad, lo que volvería a cambiar nuevamente el escenario de la política nacional. No obstante, las resoluciones de estos dilemas se presentarán solamente en el momento en que Bolsonaro y su partido estén posesionados completamente del gobierno. La única certeza que existe dentro de este contexto, es la tendencia que refleja el gobierno hacia el liberalismo en materia de economía y al conservadurismo en lo que concierne a sus principios. 


\section{Bibliografia}

Abranches, Sergio Henrique. "Presidencialismo de coalizão: O dilema institucional brasileiro". Revista de Ciências Sociais 31, No. 1 (1988): 5-34.

Amorim, Octavio. "A governabilidade sob a presidência de Lula". Revista Conjuntura Economica 57, No. 4 (2003): 22-8.

Brasil. Tribunal Superior Electoral. "Estatísticas eleitorais". TSE de Brasil. «http://www.tse. jus.br/eleicoes/estatisticas/estatisticas-eleitorais>.

__. "Partidos políticos registrados no TSE". TSE de Brasil. «http://www.tse.jus.br/partidos/partidos-politicos/registrados-no-tsè.

__ . "Repositório de dados eleitorais". TSE de Brasil. «http://www.tse.jus.br/hotsites/pesquisas-eleitorais/candidatos_anos/1989.html .

Campos, Ana Cristina. "Taxa de renovação da Câmara dos Deputados foi a maior em 20 anos". Agência Brasil, 2018.

Ferreira do Vale, Helder. "Temporality, causality and trajectories: comparative historical analysis in social and political sciences". Revista Debates 9, No. 1 (2015): 61-87.

Ferreira, Manoel Rodrigues. A evolução do sistema eleitoral brasileiro. Brasilia: Tribunal Superior Eleitoral, 2005. Edición eBooksBrasil. «ttps://bdjur.tjce.jus.br/jspui/handle/123456789/150>.

Freitas, Andréa. O presidencialismo da coalização. Rio de Janeiro: Fundação Konrad Adenauer, 2016.

Limongi, Fernando y Argelina Figueiredo. "Bases institucionais do presidencialismo de coalizão". Lua Nova, No. 44 (1998): 87-106.

Palermo, Vicente. "Brazilian political institutions: an inconclusive debate". Brazilian Political Science Review 10, No.2 (2016): 1-29.

. “Cómo se gobierna Brasil? El debate brasileño sobre instituciones políticas y gestión de gobierno". Desarrollo Económico 40, No. 159 (2000): 493-518.

Partido Social Liberal. "Brasil acima de tudo, Deus acima de todos: o caminho da prosperidade. " Propuesta de Plan de Gobierno, Brasilía, 2018.

Sartori, Giovanni. Engenharia constitucional: como mudam as constituições. Brasilia: UnB, 1996.

Shugart, Mattew y John Carey. Presidents and Assemblies: Constitutional Design and Electoral Dynamics. Cambridge: Cambridge University Press, 1992. 